\title{
6 Computational Fluid Dynamic (CFD) and Reaction Modelling Study 7 of Bio-oil Catalytic Hydrodeoxygenation in Microreactors
}

1 Received 00th January 20xx,

2 Accepted 00th January 20xx

3 DOI: $10.1039 / \mathrm{x} 0 \times x \times 00000 x$

\section{8}

19

8 Sanaa Hafeez, ${ }^{a}$ Elsa Aristodemou, ${ }^{\mathrm{a}, \mathrm{b}}$ George Manos, ${ }^{\mathrm{c}}$ S.M. Al Salem, ${ }^{\mathrm{d}}$ and Achilleas Constantinou ${ }^{* a, c, e}$

9 A Computational Fluid Dynamic (CFD) model was derived and validated, in order to, investigate the hydrodeoxygenation 10 reaction of 4-propylguaiacol, which is a lignin-derived compound present in bio-oil. A 2-D packed bed microreactor was 11 simulated using pre-sulphided $\mathrm{NiMo} / \mathrm{Al}_{2} \mathrm{O}_{3}$ solid catalyst in isothermal operation. A pseudo-homogeneous model was first 12 created to validate the experimental results from literature. Various operational parameters were investigated and validated 13 with the experimental data, such as temperature, pressure and liquid flow rate; and it was found that the CFD findings were 14 in very good agreement with the results from literature. The model was then upgraded to that of a detailed multiphase 15 configuration; and phenomena such as internal and external mass transfer limitations were investigated, as well as, reactant 16 concentrations on the rate of 4-propylguaiacol. Both models agreed with the experimental data, and therefore confirm their 17 ability for applications related to the prediction of the behaviour of bio-oil compounds hydrodeoxygenation.

\section{Introduction}

Biomass presents numerous advantages as a renewabte feedstock for bio-fuels. It contains low sulphur and nitrogen 4 and presents its self as a lucrative alternative due to its lack ${ }^{4}$ net carbon dioxide $\left(\mathrm{CO}_{2}\right)$ emissions to the environment 16 Resources from biomass consist of a vast range of materia $4 \mathrm{~S}_{6}^{7}$ such as organic waste products, forest and agricultural residues and energy crops ${ }^{2}$. Biomass feedstock's which contain cellulose hemicellulose and lignin possess a high-energy content, and 5 re often converted to oil using fast pyrolysis ${ }^{2,3}$.

Fast pyrolysis is the process of rapidly heating biomass under moderately high temperature conditions of around $500{ }^{\circ} \mathrm{C}$ and short reaction times of 2 seconds, in the absence of oxygen. In return, biomass degrades to produce mainly vapours, aerosols and some solid char. After processing, a dark brown liquid obtained which has a heating value of approximately half of tha of conventional fuel oil averaging at about $30 \mathrm{MJ} \mathrm{kg}^{-14}$. Biomas derived bio-oil has several disadvantages such as a low heating value, high viscosity and a high oxygen content, which all restrict its application as a liquid fuel. Therefore, further upgrading bio-oil by hydrodeoxygenation (HDO) is required ${ }^{5}$.

a. Division of Chemical \& Petroleum Engineering, School of Engineering, London South Bank University, London SE1 OAA, UK. Email: constaa8@Isbu.ac.uk; Tel: $+44(0) 2078157185$

b. Department of Earth Sciences, Imperial College London, London, SW7 2AZ, UK

c. Department of Chemical Engineering, University College London, London WCIE 7JE, UK.

d. Environment \& Life Sciences Research Centre, Kuwait Institute for Scientific Research, P.O. Box: 24885, Safat 13109, Kuwait.

e. Department of Chemical Engineering Cyprus University of Technology, 57 Corner of Athinon and Anexartisias, 3036 Limassol, Cyprus
41 The HDO process converts the oxygen containing compounds such as acids, aldehydes, alcohols and phenol to oxygen-free hydrocarbon fuels ${ }^{6}$. Bio-oil obtained from the fast pyrolysis of lignin contains approximately $39 \%$ of guaiacol and its derivatives. Amongst these constituents, guaiacol is often regarded as a representative model for bio-oil derived from lignin because it has two types of $\mathrm{C}-\mathrm{O}$ bonds $\left(\mathrm{Csp}_{2} \mathrm{OH}\right.$ and $\left.\mathrm{Csp}_{2} \mathrm{OCH}_{3}\right)$ within its molecular structure ${ }^{7}$. Based on this, majority of studies have utilised the compound guaiacol as a model compound and have investigated the $\mathrm{HDO}$ of guaiacol using catalysts such as $\mathrm{NiMo} / \mathrm{Al}_{2} \mathrm{O}_{3}$ and $\mathrm{CoMo} / \mathrm{Al}_{2} \mathrm{O}_{3}$, precious metal catalysts, such as platinum, ruthenium and rhodium, and nickel (Ni) catalyst ${ }^{8}$.

Phenolic compounds such as guaiacol, anisol and phenol have been extensively modelled in past studies due to their significant present in bio-oil ${ }^{6}$. Despite this, not much attention has been dedicated to lignin-derived compounds. Therefore, there exists a limited understanding of the reaction pathways and kinetics of the HDO reaction. The study of 4-propylguaiacol HDO has been selected as a basis to produce a model representing lignin-derived compounds ${ }^{3}$. 4-Propylguaiacol represents some of the key lignin-derived components present in bio-oil such as benzene, phenol, guaiacol, anisole, propyl anisole, propylphenol, and propylbenzene. The existence of phenolic compounds in the bio-oil is the origin of polymerisation and coke formation during HDO at elevated temperatures greater than $300^{\circ} \mathrm{C}^{3}$.

) Lee et al. ${ }^{8}$ studied the HDO of a model compound of ligninL derived bio-oil (guaiacol) because of its high potential to be ? used as a substitute for conventional fuels. Platinum-loaded $\mathrm{HY}$ 3 zeolites (Pt/HY) with varying Si/AL molar ratios were used as catalysts for the HDO of guaiacol, anisole, veratrole and phenol 
to a variety of hydrocarbons, such as cyclohexane. They fod $32 \mathbb{2}$ that cyclohexane was the predominant product and the $y \mathbb{E} B B$ increased with increasing number of acid sites. In orderlBAt obtain bio-oil with the maximum yield of cyclohexane alkylated cyclohexanes, the SI/AL molar ratio should $1 B 6$ adjusted to balance the Pt particle-induced hydrogenation wiBB acid site-induced methyl group transfer.

Patil et al. ${ }^{9}$ studied the HDO of the model compound guaiacoll 4 tit bio-oil to produce fuel grade oil, using a bimetal catalyst $\$ \otimes 1$ $\mathrm{Mo} / \mathrm{ZrO}_{2}-\mathrm{Al}_{2} \mathrm{O}_{3}$. The results showed that the conversion1 $\mathrm{AZ}$ guaiacol and the product yield of phenol and cyclohexane $\mathbb{1} A 3$ found to increase with increasing Mo content $(10 \%, 15 \%$, a A $20 \%)$ at continuous $\mathrm{Ni}(4 \%)$ loading and $\mathrm{Ni}$ content $(2 \%, 4 \%$, A A $6 \%)$ at continuous Mo (20\%) loading. However, there was a lower difference in guaiacol conversion and phenol and cyclohexane yield at variable $\mathrm{Ni}$ loading. Guaiacol converstan $\$$ was $100 \%$ at $330^{\circ} \mathrm{C}$ and 30 bars for the improved catalyst.

\section{7}

Taghvaei and Rahimpour ${ }^{10}$ investigated the catalytic HDO 148 guaiacol via a combination of dielectric barrier discharge (DBP and catalyst. It was found that the highest conversion 150 guaiacol (92\%) and deoxygenation degree of $65 \%$ are achieverd in the presence of $\mathrm{Pt}-\mathrm{Cl} / \mathrm{Al}_{2} \mathrm{O}_{3}$ and $\mathrm{Pt}-\mathrm{Re} / \mathrm{Al}_{2} \mathrm{O}_{3}$ catalys 55 respectively. The predominant products obtained were $\mathrm{B}^{1} \mathrm{~s}$ phenol, methylphenols and dimethylphenols. It was concluded that the difficulties of using hydrogen for the HDO reaction 155 be overcome by using the catalytic DBD reactor.

Liu et al. ${ }^{5}$ investigated the HDO of bio-oil model compounds over amorphous $\mathrm{NiB} / \mathrm{SiO}_{2}-\mathrm{Al}_{2} \mathrm{O}_{3}$ catalyst. The performancel 58 the catalysts was evaluated in an oil-water biphasic system using anisole and guaiacol as the selected model compound $\$ 95$ bio-oil. The results showed that increasing the reaction temperature or reaction time would enhance the conversion 161 guaiacol and anisole. The HDO pathways of guaiacol and anispte were studied which provided a reference for the $\mathrm{HPO}$ mechanism of bio-oil.

Microreactors present numerous advantages to investigate 160 reaction of HDO. Their enhanced surface-area-to-volume-ratif leads to a much-improved mass and heat transfer, in addition to, shorter residence time. Therefore, reactions which contaif unstable intermediates are better suited to these type of reactors because of their stability and high degree of controt $7_{1} 0$ These collective advantages of microreactors mean that thej are greener and environmentally sustainable ${ }^{12}$. In order to understand the effects of HDO on the furth 174 processing of bio-oil several studies have identiffed mathematical modelling exercises to fully simulate 1,76 interaction with compounds under various conditions ${ }^{13-16}$. FTe majority of HDO studies have focused on sulphided cobalt 178 nickel-based molybdenum (CoMo and NiMo) based catalysts $176 ?$ the separation of sulphur, nitrogen and oxygen frofif petrochemical feedstocks ${ }^{17}$.
The catalytic HDO reaction of 4-propylguaiacol to 4propylphenol using pre-sulphided $\mathrm{NiMo} / \mathrm{Al}_{2} \mathrm{O}_{3}$ catalyst is investigated and presented in this study using a packed-bed plug flow microreactor. A pseudo homogeneous model was produced, and good model validation was obtained with the experimental data. In this study, a 2-D Computational Fluid Dynamic (CFD) model was created to improve the understanding of the mass transfer and catalytic reactions taking place within the microreactor and provide an insight into any potential improvements that could be made by investigating these parameters. A validation of the model with the experimental data from literature ${ }^{3}$ is shown, and further investigations such as, internal and external mass transfer are conducted herein.

\section{Modelling Methodology}

\subsection{Reaction kinetics}

The reaction considered for the CFD mathematical modelling is the HDO of 4-propylguaiacol to 4-propylphenol (eq. 1). The kinetic studies from the experiment suggest that the kinetics for the reaction are determined by the surface reaction step which represent the competitive reaction with non-dissociative adsorption of hydrogen ${ }^{3}$. The kinetics were determined using the Langmuir-Hinshelwood-Hougen-Watson (LHHW) method, thus giving the rate equation shown below:

$\mathrm{C}_{10} \mathrm{H}_{14} \mathrm{O}_{2}+\mathrm{H}_{2} \rightarrow \mathrm{C}_{9} \mathrm{H}_{12} \mathrm{O}+\mathrm{CH}_{3} \mathrm{OH}$

$-r^{\prime}{ }_{4 P G}=\frac{k\left(K_{H 2}{ }^{e q l} C_{H 2}\right)\left(K_{4 P G}{ }^{e q l} C_{4 P G}\right)}{\left(1+K_{H 2}{ }^{e q l} C_{H 2}\right)\left(1+K_{4 P G}{ }^{e q l} C_{4 P G}\right)}$

where $k$ is the kinetic rate constant of each reaction pathway ( $\mathrm{L}$ $\mathrm{mol}^{-1}$ ) and $\mathrm{C}$ is the molar concertation at each stage of reaction mechanism. Readers are referred to Joshi and Lawal, 2013 for the reaction scheme. From the rate expression, it can be deduced that the reaction is pseudo-first-order with respect to 4-propylguaiacol and hydrogen. Table 1 displays the kinetic constants utilised for the study, and Table 2 conveys the preexponential factors, activation energies and heats of adsorption upon which the CFD modelling is based.

\subsection{Pseudo-homogeneous model}

The microreactor models were simulated using CFD to demonstrate the particle-fluid transport phenomena. Experimental work is typically costly and time-consuming, while multiphase CFD studies can deliver comprehensive information on the spatiotemporal variations in species flows, concentrations and temperatures within the reactor and with minimal effort. Therefore, CFD is a favourable approach/methodology predicting the parameters thus, enabling a detailed study of the physico-chemical processes involved ${ }^{16}$. CFD is an integrated methodology in the package that was used. 
Table 1: Reaction kinetics parameters and rate constants used as input parameters for the described model. Source: ${ }^{3}$.

193 Table 2: Reaction Pre-exponential factors, activation energy and heats of adsorption

\begin{tabular}{|c|c|c|c|c|}
\hline $\mathrm{T}\left({ }^{\circ} \mathrm{C}\right)$ & $k\left(\mathrm{~mol} \mathrm{~g}^{-1} \mathrm{~h}^{-1}\right)$ & $\mathrm{K}_{4 \mathrm{PG}}\left(\mathrm{L} \mathrm{mol}^{-1}\right)$ & $\mathrm{K}_{\mathrm{H} 2}\left(\mathrm{~L} \mathrm{~mol}^{-1}\right)$ & $\mathbf{R}^{2}$ \\
\hline 250 & $22.21 \pm 0.77$ & $0.02 \pm 0.002$ & $0.3 \pm 0.004$ & 0.97 \\
\hline 300 & $41.40 \pm 4.21$ & $0.1 \pm 0.01$ & $0.1 \pm 0.01$ & 0.96 \\
\hline 350 & $77.78 \pm 0.01$ & $0.6 \pm\left(9.7 \times 10^{-5}\right)$ & $0.02 \pm\left(1.6 \times 10^{-6}\right)$ & 0.96 \\
\hline
\end{tabular}

\section{1}

222

\section{Intrinsic constant} Pre-exponential factors

$k_{0}\left(\times 10^{4} \mathrm{~mol} \mathrm{~g}^{-1} \mathrm{~h}^{-1}\right)$

$K_{4-\text { Propylguaiacol, } 0}\left(\times 10^{7} \mathrm{~L} \mathrm{~mol}^{-1}\right)$

$K_{H 2,0}\left(\times 10^{-8} \mathrm{~L} \mathrm{~mol}^{-1}\right)$

\section{Activation energies and heats of adsorption}

\section{$E_{a}\left(\mathrm{~kJ} \mathrm{~mol}^{-1}\right)$}

$\Delta H_{4-\text { Propylquaiacol }}\left(\mathrm{kJ} \mathrm{mol}^{-1}\right)$ $\Delta H_{H 2}\left(\mathrm{~kJ} \mathrm{~mol}^{-1}\right)$

$33.86 \pm 2.70$

$91.85 \pm 2.69$

$-72.69 \pm 2.63$

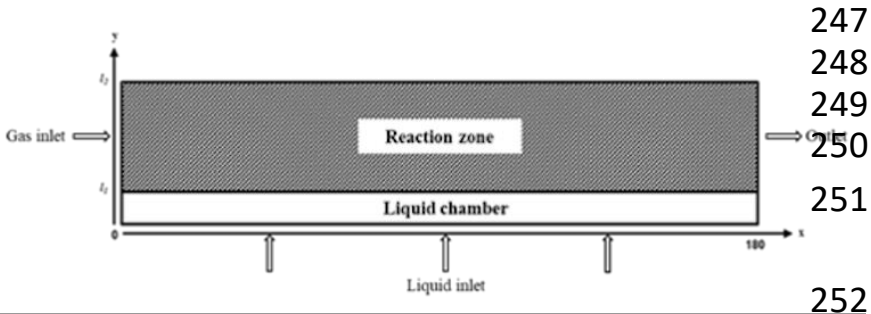

Figure 1: Microreactor model used for the CFD simulation. Note to readers: 453 schematic is not to scale.

223

224

225

226

227

228

A 2-D microreactor model was created based on the assumption that the concentration and temperature gradients occur only 233 the axial direction. The only transport mechanism operating in this direction is the overall flow itself, and this is of plug flow type. Further assumptions which the model was founded upo 36 are (a) Application of steady-state and isothermal conditions; (b) there is a pressure drop of $0.07 \mathrm{MPa}$ along the length of $\mathrm{ZB} \mathrm{B}$ microreactor; (c) Henrys law applies is valid for the gas-liq 2388 interface; (d) the ideal gas law applies for the fluids in the $\mathbf{z} 3 \mathbf{3}$ phase; and that (d) there is a constant axial fluid velocity in $2 / 40$ gas phase with uniform physical properties and transp $24 \mathbb{4}$ coefficients. The microreactor has a height of $0.762 \mathrm{~mm}$, and a length of $180 \mathrm{~mm}$. The catalyst used is a pre-sulphided $\mathrm{NiMo} / \mathrm{Al} 2 \mathrm{O} 3$ in the form of solid spherical particles. Figure 1 shows a schematic diagram of the model used for $\$ 43$ simulation.

\subsection{Mass balances in microreactor}

Modelling of the liquid chamber was established by connecting the gas phase reaction zone with the bulk liquid phase. The mass balance equation for the 4-propylguaiacol in the liquid phase is expressed as:

$u_{x} \frac{\delta c_{4 P G}}{\delta x}=D_{i}\left(\frac{\delta^{2} c_{4 P G}}{\delta x^{2}}+\frac{\delta^{2} c_{4 P G}}{\delta y^{2}}\right)$

where $c_{4 P G}$ is the concentration of 4-propylguaiacol in the bulk liquid phase in $\mathrm{mol} / \mathrm{m}^{3}, D_{i}$ is the molecular diffusion coefficient $(\mathrm{m} / \mathrm{s})$ in the bulk liquid, $u_{x}$ is the liquid velocity in the axial direction.

The mass balance in the gas phase reaction zone is expressed as:

$D_{i}\left(\frac{\delta^{2} c_{i}}{\delta x^{2}}+\frac{\delta^{2} c_{i}}{\delta y^{2}}\right)+u_{x} \frac{\delta c_{i}}{\delta x}=R$

where $i$ represents 4-propylguaiacol or $\mathrm{H}_{2}$, and $R$ incorporates the rate of reaction term. The molecular diffusion coefficient of 4-propylguaiacol $\left(D_{4 P G}\right)$ in the fluid was calculated using the Reddy-Doraiswamy correlation ${ }^{18}$.

$D_{4 P G}=1 \times 10^{-16}\left(\frac{T \sqrt{M_{4 P G}}}{\mu V_{4 P G}^{\frac{2}{3}}}\right)$

where $T$ is the temperature in $\mathrm{K}, M_{4 P G}$ is the molecular mass of 4-propylguaiacol in $\mathrm{g} / \mathrm{mol}, \mu$ is the viscosity of 4-propylguaiacol in $\mathrm{Pa} \cdot \mathrm{S}$ and $V_{4 P G}$ is the molar volume of 4-propylguaiacol at normal boiling point in $\mathrm{m}^{3} / \mathrm{kmol}^{19}$. The molecular diffusion coefficient of hydrogen $\left(D_{\mathrm{H}_{2}}\right)$ was obtained using the WilkeChang correlation ${ }^{20}$.

$D_{H_{2}}=1.1728 \times 10^{-16} \frac{T \sqrt{\chi M_{4 P G}}}{\mu V_{H_{2}}^{0.6}}$

where $\chi$ is the association factor of 4-propylguaiacol, 1 for nonassociated solvents, $V_{H_{2}}$ is the molar volume of hydrogen at normal boiling point in $\mathrm{m}^{3} / \mathrm{kmol}^{21}$. The boundary conditions 
at $y=0$;

$c_{4 P G}=c_{4 P G, i n,}$

$$
c_{\mathrm{H}_{2}}=0
$$

The mass balance equations coupled with the approprBO9 boundary conditions were solved using COMSOL Multiphys $32 \$ 0$ software version 5.3. The finalized geometry comprised of 1 al mesh consisting of 4400 domain elements and 400 bound 312 elements, and 9,090 degrees of freedom was used, and BHe3 results were found to be mesh independent with31af computational time of 5 seconds.

\subsection{Detailed multiphase model}

The previous pseudo-homogeneous model shown in section 31.8 was further enhanced to incorporate the catalyst particles 319 which the gas phase reaction occurs. The assumptions for 3120 pseudo-homogeneous model are applicable to this detaßed model, with the exception that the reaction zone in figure 1 is packed with solid spherical catalyst particles of the same and shape. The mass balance equation for the species in 8123 catalyst bed is expressed as:

$u_{x} \frac{\delta c_{i}}{\delta x}=D_{i, A} \frac{\delta^{2} c_{i}}{\delta x^{2}}+D_{i, T} \frac{\delta^{2} c_{i}}{\delta y^{2}}-J_{i} S_{b}$

$301 J_{i}=h_{i}\left(c_{i}-c_{i, p s}\right)$

where, $\varepsilon$ is the fractional voidage of the packed bed and $S$ is $B B E$ specific surface area, in $m$, of the particles. For spher 3336 particles this is given by:

where, $r_{p}$ is the catalyst particle radius.

340

An assumption of the film condition is realised at the pellet-fbuid interface. The mass flux across this pellet-fluid interface into the pellet is potentially rate determined by the resistance to $\mathbf{m}^{2} \$ 2$ transfer on the bulk fluid side. This resistance can be expressed in terms of the external mass transfer coefficient:
$h_{i}=\frac{S h \cdot D_{i}}{2 r_{p}}$

$S h=2+0.552 R e^{1 / 2} S c^{1 / 3}$ where, $c_{i, p s}$ is the concentration of $i$ at the catalyst particle
surface and $h_{i}$ is the external mass transfer coefficient. $S c$ is the surface and $h_{i}$ is the external mass transfer coefficient. $S c$ is the
Schmidt number, $\mu$ is viscosity of 4-propylguaiacol and $\rho$ is the density of 4-propylguaiacol. $R e$ is the particle Reynolds number ${ }^{23}$. Sh is the Sherwood number, which is based upon the Frössling correlation ${ }^{24}$.

The chemical reaction which occurs inside (within) the pellets is incorporated into the mass balances with the Reactive Pellet Bed feature in $\mathrm{COMSOL}^{\circ}$. This feature has a predefined 1-D extra dimension on the normalised radius of the catalyst pellet particle $\left(r=r_{\text {dim }} / r_{p e}\right)$. The mass balance inside the catalyst pellet is obtained by performing a shell balance across a spherical shell:

$\frac{\delta}{\delta r}\left(r^{2} D_{i, e f f} \frac{\delta c_{i, p}}{\delta r}\right)=r^{2} r_{p} R_{i, p}$

where $r$ is the catalyst particle radius (dimensionless), $D_{i, \text { eff }}$ is the effective diffusion coefficient of chemical species $i$ in the catalyst pores, $c_{i, p}$ is the concentration of chemical species $i$ in the catalyst particle in $\mathrm{mol} / \mathrm{m}^{3} . R_{i, p}$ is the reaction source term (rate of reaction per unit volume of catalyst particle). The effective diffusivities of 4-propylguaiacol and hydrogen into the pores of the catalyst pellet are calculated by relating the diffusion coefficient to either the bulk or Knudsen diffusivity.

$D_{i, e f f}=\frac{D_{i, A B} \phi_{p} \sigma_{c}}{\tau}$

where, $D_{i, A B}$ is the bulk diffusivity of chemical species $i, \Phi_{p}$ is the pellet porosity, $\sigma_{c}$ is the constriction factor and $\tau$ is the tortuosity. Typical values of the constriction factor, the tortuosity, and the pellet porosity are, respectively, $\sigma_{c}=0.8, \tau=$ 3.0 and $\Phi_{p}=0.4^{25}$. Boundary conditions used were as per the following:

at $x=0 ; \quad c_{H_{2}}=c_{H_{2}, i n}, c_{4 P G}=0$

at $y=I_{1} ; \quad c_{4 P G, b}=H^{*} c_{4 P G}, \frac{\delta c_{H 2}}{\delta y}=0$

at $x=180, \frac{\delta c_{i}}{\delta x}=0$

at $y=0 ; \quad C_{4 P G}=C_{4 P G, i n}, C_{H_{2}}=0$

343 at $r=1 ; c_{i, p}=c_{i, p s}$

344 at $r=0 ; \frac{\delta c_{i, p}}{\delta r}=0$ 
Table 3: The list of parameters used for the CFD models

347

\begin{tabular}{|c|c|c|c|c|}
\hline Symbol & Value & Units & Description & \\
\hline $\mathrm{C}_{4 \mathrm{PG}, \mathrm{in}}$ & 1.1 & $\mathrm{~mol} \mathrm{~m}^{-3}$ & 4-propylguaiacol inlet concentration & \\
\hline $\mathrm{C}_{\mathrm{H} 2, \mathrm{~g}}$ & $\frac{\mathrm{P}_{\mathrm{H} 2}}{\mathrm{RT}}$ & $\mathrm{mol} \mathrm{m}^{-3}$ & Hydrogen concentration in the gas phase & \\
\hline$u$ & $0.03-0.18$ & $\mathrm{~mL} \min ^{-1}$ & 4-propylguaiacol inlet flow rate & \\
\hline $\mathrm{F}_{\mathrm{H} 2 \text {,in }}$ & $30-120$ & $\mathrm{~cm}^{3} \mathrm{~min}^{-1}$ & Hydrogen inlet flow rate & \\
\hline $\mathrm{H}$ & $\mathrm{I}_{1}+\mathrm{I}_{2}$ & $\mathrm{~mm}$ & Reactor height & \\
\hline W & 180 & $\mathrm{~mm}$ & Reactor width & \\
\hline $\mathrm{I}_{2}$ & 0.762 & $\mathrm{~mm}$ & Reaction zone height & \\
\hline $\mathrm{l}_{1}$ & $0.762 \times 10^{-3}$ & $\mathrm{~mm}$ & Liquid chamber height & \\
\hline $\mathrm{D}_{4 \mathrm{PG}}$ & $9.995 \times 10^{-8}$ & $\mathrm{~m}^{2} \mathrm{~s}^{-1}$ & $\begin{array}{l}\text { Diffusion coefficient of 4-propylguaiacol in the bulk } \\
\text { fluid }^{18,19}\end{array}$ & \\
\hline $\mathrm{D}_{\mathrm{H} 2}$ & $1.23 \times 10^{-8}$ & $\mathrm{~m}^{2} \mathrm{~s}^{-1}$ & Diffusion coefficient of hydrogen in the bulk fluid 15 & \\
\hline $\mathrm{D}_{4 \mathrm{PG}, \mathrm{eff}}$ & $1.312 \times 10^{-9}$ & $m^{2} s^{-1}$ & $\begin{array}{l}\text { Effective diffusion coefficient of 4-propylguaiacol in } \\
\text { the catalyst particle } 25\end{array}$ & \\
\hline $\mathrm{D}_{\mathrm{H} 2, \mathrm{eff}}$ & $1.066 \times 10^{-9}$ & $m^{2} s^{-1}$ & $\begin{array}{l}\text { Effective diffusion coefficient of hydrogen in the } \\
\text { catalyst particle }{ }^{25}\end{array}$ & \\
\hline$\rho_{b}$ & 580 & $\mathrm{~kg} \mathrm{~m}^{-3}$ & Density of catalyst bed & \\
\hline$\rho_{\mathrm{pe}}$ & 750 & $\mathrm{~kg} \mathrm{~m}^{-3}$ & Density of catalyst pellet & \\
\hline$\varepsilon_{\mathrm{pe}}$ & 0.4 & - & Porosity of pellet & \\
\hline$\varepsilon_{\mathrm{b}}$ & $1-\rho_{b} / \rho_{p e}$ & - & Void fraction of catalyst bed & \\
\hline$r_{\mathrm{pe}}$ & $1.1 \times 10^{-4}$ & $\mathrm{~m}$ & Radius of catalyst pellet & \\
\hline $\mathrm{R}$ & $8.314 \times 10^{-9}$ & $\mathrm{~m}^{3} \cdot \operatorname{bar}\left(\mathrm{K}^{-1} \mathrm{~mol}^{-1}\right)$ & Ideal gas constant & \\
\hline $\mathrm{M}_{4 \mathrm{PG}}$ & 166.22 & $\mathrm{~g} \mathrm{~mol}^{-1}$ & Molecular weight of 4-propylguaiacol & \\
\hline
\end{tabular}

The mass balance equations coupled with the appropriate boundary conditions were solved using COMSOL Multiphysics ${ }^{\circ}$ software version 5.3. The finalized geometry comprised of a mesh consisting of 440,000 domain elements and 30,088 boundary elements, and 106,858 degrees of freedom was used, and the results were found to be mesh independent with a computational time of 12 seconds. The variables, which were used to solve the models, are shown in table 3.

\section{Results and Discussion}

\subsection{Model validation}

\subsubsection{Effects of temperature}

The results obtained from the CFD pseudo homogeneous alpd detailed models were compared to the experimental data. Bqth models were compared to experimental results for 424 microreactor operating at a pressure of 300 psig, 21245 temperatures of $250-450{ }^{\circ} \mathrm{C}$. The comparison between 426 simulated results and the experimental results give 427 indication towards the validity and robustness of the model 8 Figure 2 shows the comparison between the modelling $2_{2} 29$ experimental results. The graph depicts the conversion of 40 propylguaiacol obtained for a temperature range of 250-45939 with a good agreement between the experimental and obtainech CFD (i.e. pseudo-homogeneous and detailed model) results. 433

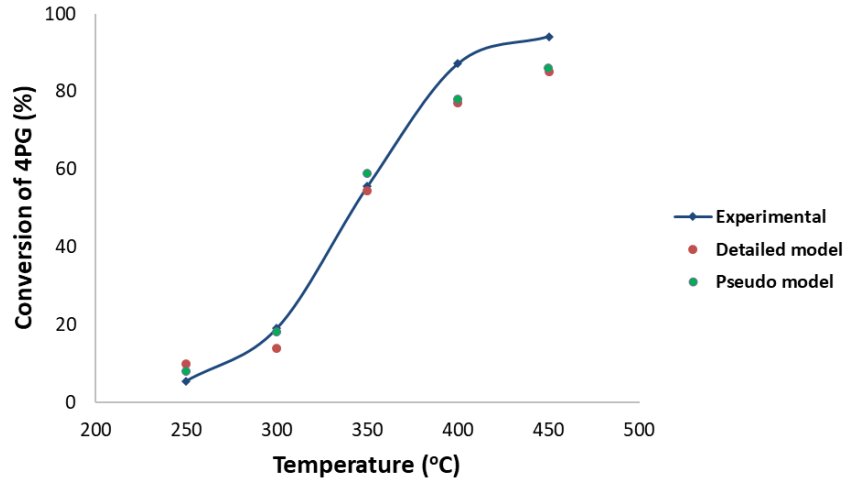

Figure 2: Comparison between model predicted results and experimental results: Pressure, 300 psig; gas phase, hydrogen; liquid phase, 4-propylguaiacol.

The results show that as the temperature increases, the conversion of 4-propylguaiacol also increases. For temperatures below $400{ }^{\circ} \mathrm{C}$, there is a very good validation between the results; however, there is a slight deviation in the results at temperatures, which exceed $400^{\circ} \mathrm{C}$. This could be due to the fact that secondary side reactions are occurring within the microreactor ${ }^{26}$, these reactions are not considered within the CFD modelling due to the lack of reaction kinetic data available. The predominant products from the HDO of 4-propylgauaicol using the presulfided-NiMo/ $/ \mathrm{Al}_{2} \mathrm{O}_{3}$ catalyst are 4-propylphenol, 4-ethylphenol, 4-propylbenzene, phenols and cresols with insignificant quantities of benzene and toluene. The compound 4-propylphenol is solely brought to attention in this study because it has the highest selectivity. 


\subsubsection{Effects of hydrogen partial pressure}

The effect of hydrogen partial pressure on the conversion o $\$ 80$ propylguiacol was investigated using the models develope $\$ 1$ this work and were compared with the results from literat 4 \& 82 The range of pressures used for the study varied from $240-483$ psig at a constant reactor temperature of $400{ }^{\circ} \mathrm{C}$ with a const $48 \mathrm{~A}$ residence time. The results in Figure 3 show that as 4185 hydrogen partial pressure increases, the conversion of 486 propylguaiacol also increases. However, at pressures 487 approximately $400 \mathrm{psig}$, the conversion remains relati4 88 constant. This is because at higher temperatures there 48 maximum adsorption of hydrogen on the surface of the cataAf 80 resulting in a stable conversion. The comparison in res4QA工 between the CFD modelling and experimental show a very gA\&2 agreement.

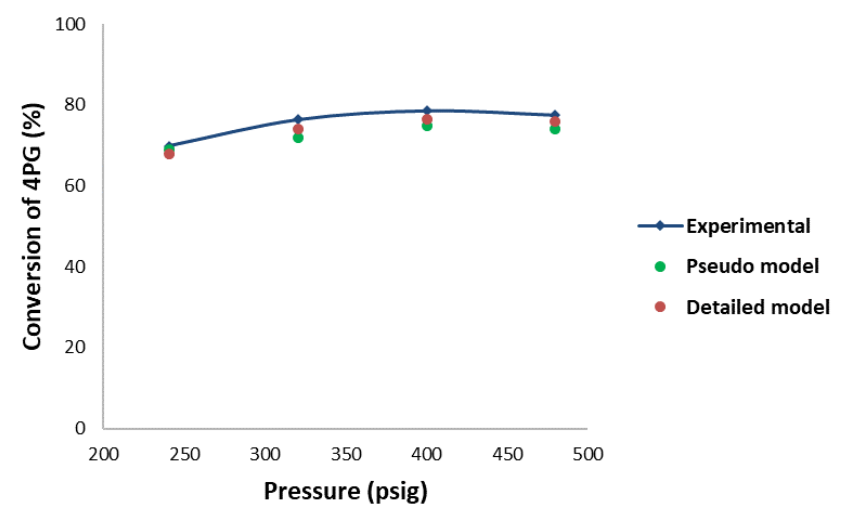

Figure 3: Effect of hydrogen inlet pressure on the conversion of 4-Propylguaiacol: Temperature, $400{ }^{\circ} \mathrm{C}$; gas phase, hydrogen; liquid phase 4-propylguaiacol,4g6 phase, hydrogen.

\subsubsection{Effects of liquid flow rate of 4-propylguaiacol}

499

The effect of liquid flow rate on the conversion of 4propylguaiacol was also studied using the proposed models. The liquid flow rate of 4-propylguaiacol varied between 0.03 $0.15 \mathrm{~mL}$ min-1, and the remaining operating parameters were kept constant. The studies were performed at a temperature and pressure of $400^{\circ} \mathrm{C}$ and 300 psig respectively. Figure 4 shows that as the liquid flow rate increases, the conversion of 4propylguaiacol decreases. The reacting fluids spend a shorter time within the microreactor as the liquid flow rate decreases, hence the conversion declines. Both models predict a decreasing conversion profile with the liquid flow rate which is consistent with the dependence of conversion of a reactant upon space velocity. 4-propylguaiacol is only a reactant in the reaction network presented by the experimentalists ${ }^{3}$, who 1 cannot justify the existence of a maximum in the profile. Th ${ }_{3} \mathrm{C}_{3}$ also consistent with the findings and conclusions of 5103 experimentalists $^{3}$, who also describe their experime 5 it 4 conversion profile as decreasing. We believe that the maximson 5 in the experimental profile is an artefactual one and causect 506 small experimental errors. The deviation of the experimentat conversion values is within in the experimental error range. 508 509
As the results obtained from the models are in agreement with the experimental findings ${ }^{3}$, they demonstrate a good validation of our models.

Figure 5 shows the concentration profiles of 4-propylguaiacol in the bulk fluid phase across the transverse direction at different axial positions. It can be observed that the concentration of 4propylguaiacol is highest at the initial axial positions of the microreactor, this is where the 4-propylguaiacol first encounters the hydrogen at the interface ( $a t x=0 ; y=0)$. As the axial position of the microreactor progresses, the concentration decreases until eventually becoming stable. This is because, as the concentration of hydrogen increases along the axial direction of the microreactor, it will encounter greater concentrations of the 4-propylguaiacol, which results in a stable reactant conversion.

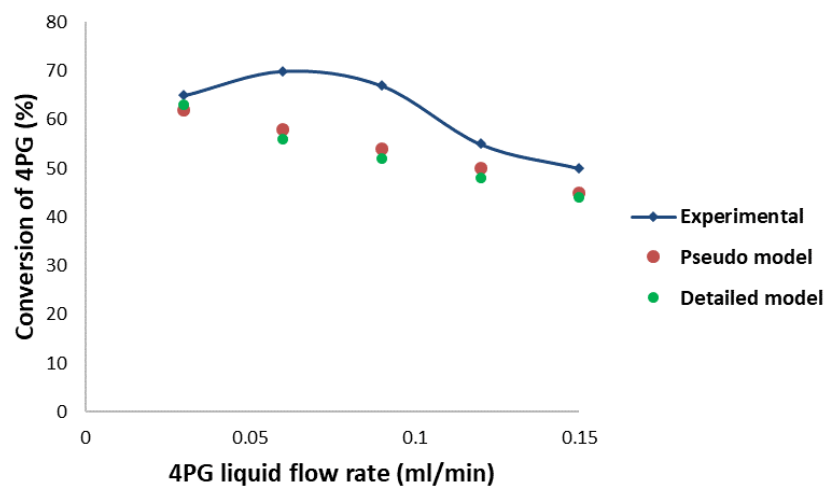

Figure 4: Effect of 4-propylguaiacol liquid flow rate on the conversion of 4 Propylguaiacol: Temperature, $400{ }^{\circ} \mathrm{C}$; pressure, 300 psig; gas phase, hydrogen; liquid phase 4-propylguaiacol.

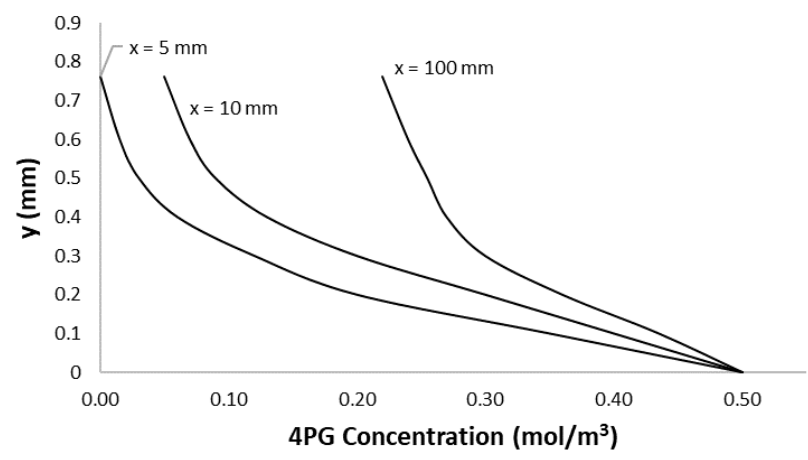

Figure 5: Concentration profiles of 4-propylguaiacol in the bulk fluid phase at a constant transverse direction and varying axial positions: Temperature, $400{ }^{\circ} \mathrm{C}$; pressure, 300 psig; gas phase, hydrogen; liquid phase 4-propylguaiacol.

\subsection{Kinetic studies}

\subsubsection{Rate analysis}

The effect of hydrogen concentration on the rate of disappearance of 4PG was investigated using the detailed model. The hydrogen concentration varied between $0-0.15$ $\mathrm{mol} / \mathrm{L}$, and the concentration of 4-propylguaiacol remained 
510 constant at $1.1 \mathrm{~mol} / \mathrm{L}$. Three different temperatures of 350542 $511300{ }^{\circ} \mathrm{C}$ and $250{ }^{\circ} \mathrm{C}$ were investigated to obtain the $5 \mathrm{AB}$ 512 modelling results. All other parameters were kept constant, 5iAdA 513 the conversion of the reactants was limited to a maximun $\overline{ } \mathbf{} \mathbf{} \overline{5}$ $51410 \%$ in order to solely determine the reaction rates foundec5 46 515 the initial concentrations of the reacting fluids. Figure 6 sh 547 516 the results obtained from the study. It can be observed thaБ 48 517 the concentration of hydrogen increases, the rate of549 518 propylguaiacol also increases.

The effect of 4-propylguaiacol concentration on the rate $52 \mathbb{2}$ disappearance of 4-propylguaiacol was then investigated. $\$ 58$ 4-propylguaiacol concentration varied from 0.1-2.1 mol/L, 5 554 constant hydrogen pressure of $208 \mathrm{psig}$. The th555 temperatures used for the study were $350{ }^{\circ} \mathrm{C}, 300^{\circ} \mathrm{C}$ and 856 ${ }^{\circ} \mathrm{C}$. Figure 7 shows the results obtained. It can be observed $\$ 55 \mathrm{t}$ at lower concentrations of 4-propylguaiacol the rate of5 $\$ 8$ propylguaiacol appears to increase. However, at hig 59 concentrations, the rate remains relatively constant.

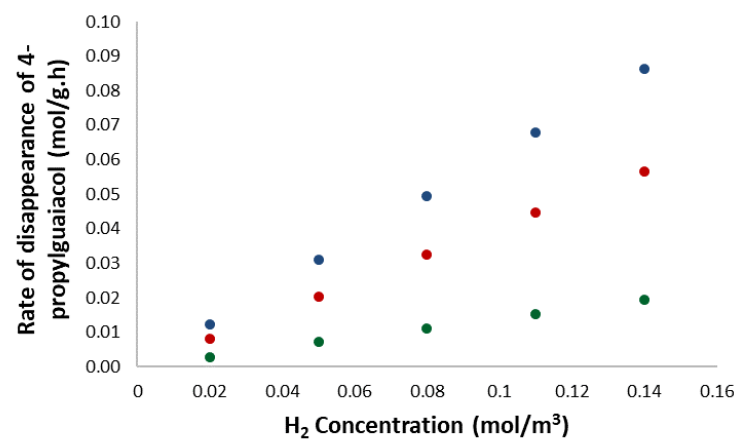

561. !

529 531

Figure 6: Effect of hydrogen concentration on the rate of 4-Propylguaiacol 571 phase, hydrogen; liquid phase 4-propylguaiacol.

572 573 571

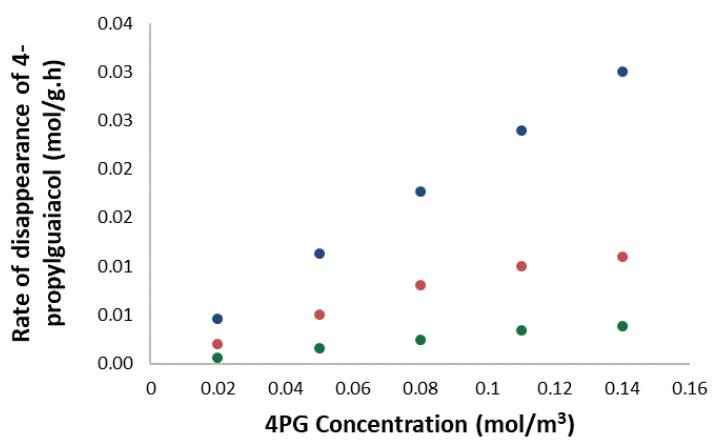

Figure 7: Effect of hydrogen concentration on the rate of 4-Propylguaiacol: Gas phase, hydrogen; liquid phase 4-propylguaiacol.

\subsection{Internal and External mass transfer limitations} catalyst, and so it can ascertain the internal and external me80 transfer resistances within the microreactor. The model $5 \mathbb{8} 1$ demonstrate which parameters lead to the reaction bejigg diffusion limited or surface-reaction-limited. Using the $\overline{5} 883$ models to study the internal mass transfer limitations can al 15084 the determination of which factors enhance or diminish the mass transfer rates (affecting the apparent rate of reaction); therefore, an understanding of the optimisation of the HDO reaction can be achieved.

\subsubsection{Internal mass transfer limitations}

The concentration profiles of 4-propylguaiacol are presented in figure 8 . The profiles were obtained at $x=90 \mathrm{~mm}$, and varying reactor heights of $y=0.7 ; 0.5$ and $0.2 \mathrm{~mm}$, for a catalyst particle size of $75-150 \mu \mathrm{m}$. The internal mass transfer resistance is responsible for the concentration gradient within the catalyst particle. It can be deduced that the difference in concentration from the surface of the particle, to within the particle, is less than $5 \%$. In addition, the model was simulated with constant reactor properties, and catalyst particle sizes which were halved and quartered. It was observed that there was no significant difference in the conversion of 4-propylguaiacol (less than $2 \%$ ). The internal mass transfer limitation was further validated by calculating the Thiele modulus $(\Phi)$ for a particle size ranging from $70-160 \mu \mathrm{m}$ by assuming the reaction to be pseudo-firstorder with respect to 4-propylguaiacol and hydrogen ${ }^{25}$.

$\Phi=\frac{r_{p}}{3}\left(\frac{\rho_{p} \cdot\left(-r^{\prime}{ }_{4 P G}\right)}{D_{e} \cdot C_{H 2}}\right)^{\frac{1}{2}}$

where $\rho_{\mathrm{p}}$ is the density of the catalyst pellet. The Thiele modulus was found to be approximately 0.5 . For this value of the Thiele modulus, the effectiveness factor is found to be 1 ; which suggests that the reaction is surface-reaction-limited (overall rate of reaction is equal to the rate of reaction obtained from within the catalyst particle maintaining the same conditions as the particle surface). Therefore, from this study, it can be concluded that there is negligible internal mass transfer resistance. This conclusion coincides with that obtained from the experimental results.

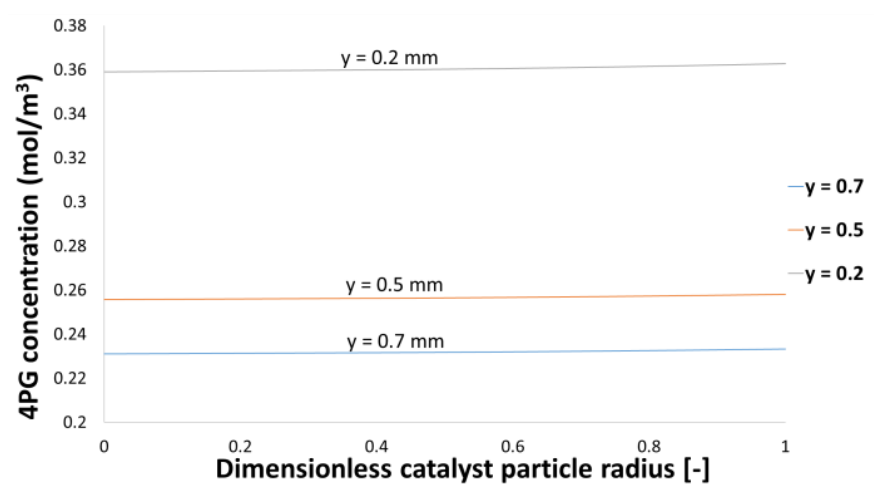

Figure 8: Concentration of 4-Propylguaiacol within the catalyst particles at $x=90$ $\mathrm{mm}$, generated from modelling: Gas phase, hydrogen; liquid phase 4propylguaiacol; reaction temperature $400^{\circ} \mathrm{C}$.

The size of the catalyst particle was doubled and quadrupled to introduce pore diffusion limitations. Figure 9 shows the concentration profiles obtained at $x=90 \mathrm{~mm}$, and varying reactor heights of $y=0.7 ; 0.5$ and $0.2 \mathrm{~mm}$. The introduction of the intraparticle transport resistances is responsible for the 


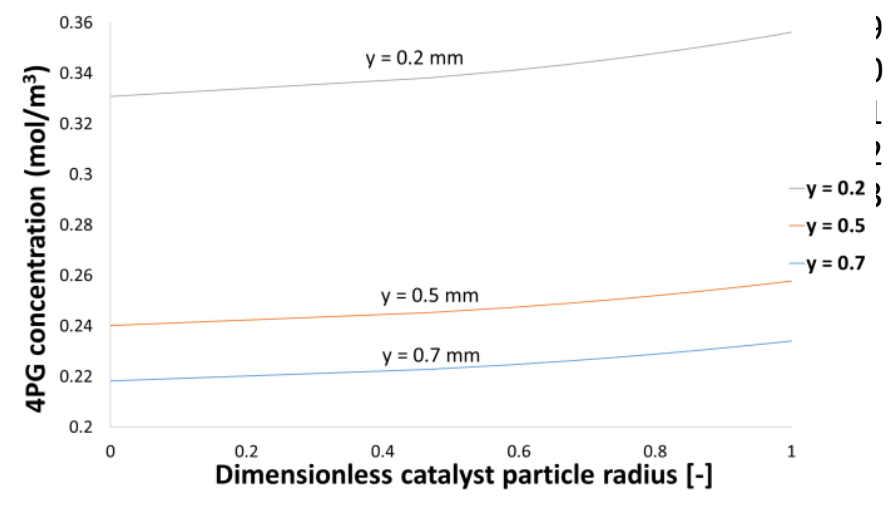

Figure 9: Concentration of 4-Propylguaiacol within the catalyst particles at $\mathrm{x}=90$ $\mathrm{mm}$, generated from modelling: Gas phase, hydrogen; liquid phase 4propylguaiacol; reaction temperature $400{ }^{\circ} \mathrm{C}$.

$604 \eta=\frac{3}{\Phi^{2}}(\Phi \operatorname{coth} \Phi-1)$

The comprehensive heterogeneous model was used to obtain the internal effectiveness factor. This provides an indication of the relative importance of diffusion and reaction limitations. The effectiveness factor is regarded as the ratio between 924 actual overall rate of reaction, and the rate of reaction the would occur if the interior surface of the pellet were expose $\$ 29$ the external surface conditions ${ }^{25}$. The effectiveness factor for28 spherical catalyst particle following a first-order reaction car 6 beg obtained as:

$$
\eta=\frac{3}{\Phi^{2}}(\Phi \operatorname{coth} \Phi-1)
$$

Figure 10 shows a plot of the effectiveness factor against 6 Be4 Thiele Modulus using the detailed model. For the catalyst peA3Б sizes between $75-150 \mu \mathrm{m}$, the Thiele Modulus is approxima6e36 less than 1 , this corresponds to an effectiveness factor of u6iB which suggests negligible internal mass transfer resistan 638 However, increasing the particle sizes results in a decreas $63 \theta$ the effectiveness factor, suggesting that the reaction640 becoming diffusion-limited within the pellet.

\section{1}

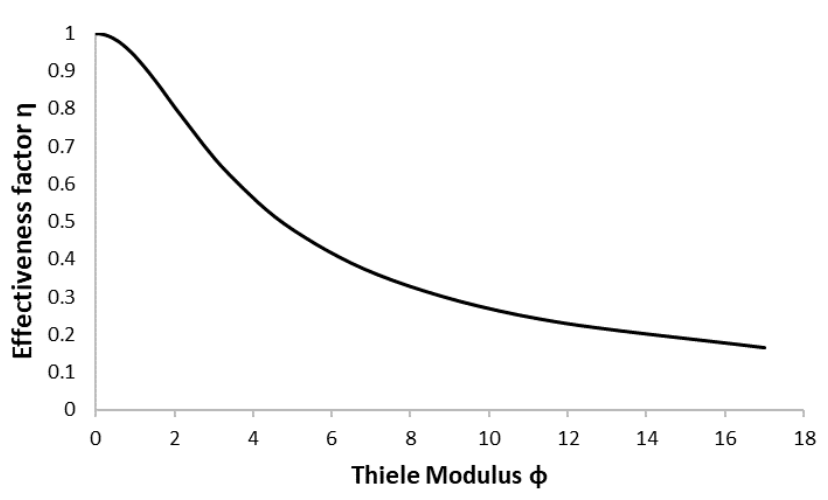

613
Figure 11 shows a plot of the conversion of 4-propylguaiacol and the liquid flow rate for the pseudo-homogeneous model, detailed model and the detailed model with a catalyst size of $220 \mu \mathrm{m}$. It can be observed that the reactant conversion decreases with the larger catalyst sizes. From the modelling results, the overall rate of reaction could be enhanced by decreasing the catalyst particle size; increasing the internal surface area; increasing the temperature; and increasing the concentration.

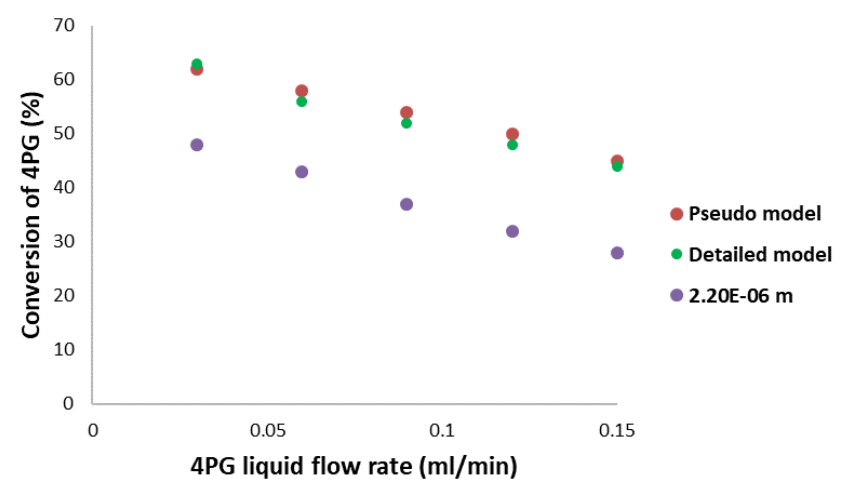

Figure 11: Effect of 4-propylguaiacol liquid flow rate on the conversion of 4 propylguaiacol for different model configurations: Temperature, $400{ }^{\circ} \mathrm{C}$; pressure, 300 psig; gas phase, hydrogen; liquid phase 4-propylguaiacol.

A study of comparison between the pseudo-homogeneous and detailed model was conducted. Figure 12 shows the conversion of 4-propylguaiacol against the liquid flow rate for the detailed model, comprising of catalyst particle sizes ranging between 220-500 $\mu \mathrm{m}$, and the pseudo-homogeneous model. The results show that as the pellet sizes increase, the reactant conversion decreases because of the diffusion limitations within the particle. The reaction rates obtained from the pseudohomogeneous model were multiplied with the effectiveness factor to obtain the results shown in figure 12. There is a good agreement between the results which demonstrates the validity and robustness between the two models. As a result, either model can be utilised to demonstrate the catalytic hydrodeoxygenation of bio-oil to produce desirable results.

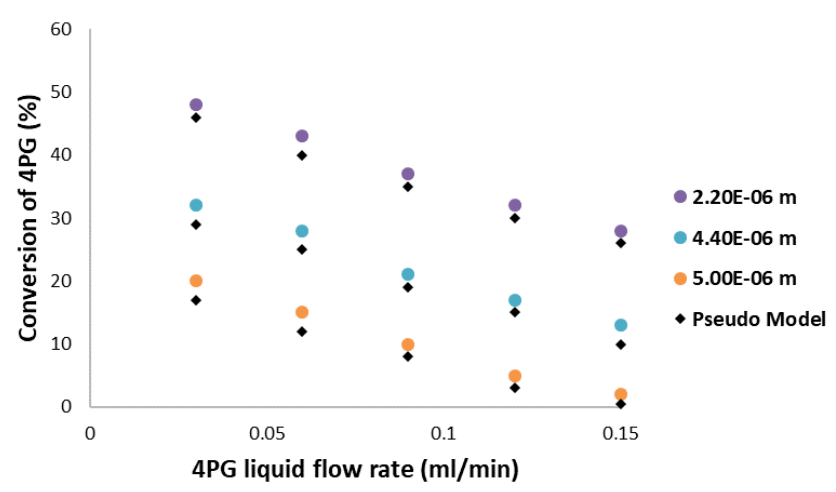

Figure 12: Effect of 4-propylguaiacol liquid flow rate on the conversion of 4 propylguaiacol for different catalyst pellet sizes: Temperature, $400{ }^{\circ} \mathrm{C}$; pressure, 300 psig; gas phase, hydrogen; liquid phase 4-propylguaiacol. 


\subsubsection{External mass transfer limitations}

The external mass transfer resistance was then investigate 87 The hydrodeoxygenation reaction involves the diffusion 6088 mass transfer of hydrogen gas into the 4-propylguaiacol liceinet phase, and subsequently diffusion through the liquid phas $\$ 00$ the proximate area of the catalyst particle. In order601 investigate the resistance to the diffusion across the bound 692 layer, the concentration surrounding the catalyst part 693 should be compared to the concentration on the surface of 6194 particle. Figure 13 shows the bulk concentrations of 695 propylguaiacol compared to the concentrations obtained fi 696 the surface of the catalyst particle. The concentrations o697 propylguaiacol in the bulk are found to be less than $2 \%$ w6ê 8 compared to the concentrations on the surface of the cata 698 particle. It can be deduced that there is a negligible resista $\mathrm{R}_{\mathrm{O}} \mathrm{Q}$ to mass transfer, and this agrees with the experimental res $\mathbb{R}$, thy obtained from Joshi and Lawal (2013).

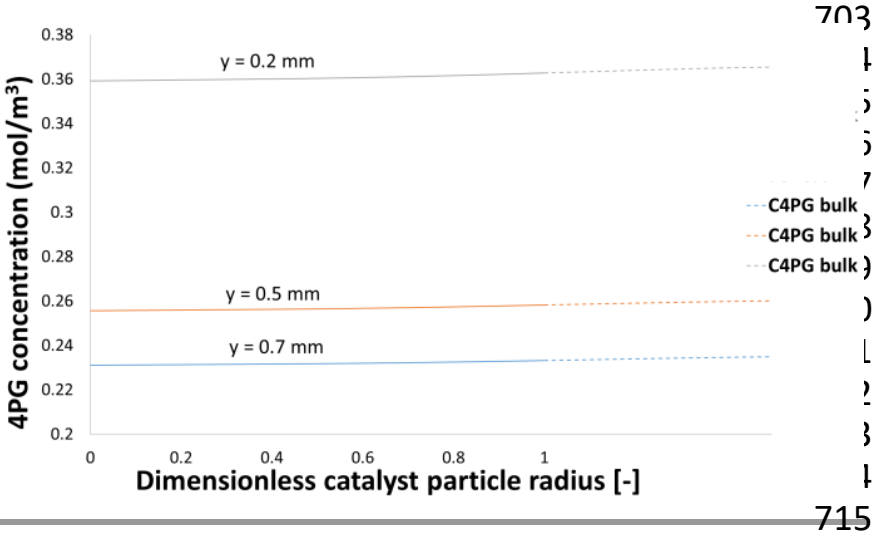

Figure 13: Comparison between concentration of 4-Propylguaiacol within7tin6 catalyst particles and in the bulk at $x=90 \mathrm{~mm}$, generated from modelling:-9095 phase, hydrogen; liquid phase 4-propylguaiacol; reaction temperature $400{ }^{\circ} \mathrm{C}$.

\section{Conclusions}

720

666
The modelling results obtained from the CFD study for 721 catalytic HDO of 4-propylguaiacol to 4-propylphenol haye demonstrated a sound validation with the experimental data obtained from literature ${ }^{3}$. The slight deviations in results at temperatures greater than $350^{\circ} \mathrm{C}$ could owe to the fact that the results closely follow the \% yield of other major reactiog 25 products. The secondary side reactions are not considered in this work (due to the lack of kinetic data available). Therefore, the models developed could have slight limitations at higher temperatures because of the previously mentioned reasons; however, this would be overcome by the development of more complex kinetic models which account for the side reaction 8 interacting with the HDO of 4-propylguaiacol to 4-propylphe\#ə29 Further model validations were demonstrated by the effects of pressure and liquid flow rate illustrate a good validation with the experimental data. It can be observed that for this spedipe reaction, the conversion of 4-propylguaiacol increased wigh 1 increasing temperature and pressure; however, increasing $\neq 132$ liquid flow rate appears to decrease the reactant conversion. 73
The detailed model further allowed the investigation of the concentrations of hydrogen and 4-propylguaiacol on the rate of 4-propylguaiacol. The model results demonstrated that as the concentration of these reactants increased, the rate of disappearance of 4-propylguaiacol also increased. In addition, the detailed model gave rise to the characterisation and evaluation of the reaction-coupled transport phenomena taking place within the catalyst bed in the microreactor. The internal and external mass transfer limitations were investigated by obtaining concentration profiles within the individual catalyst particle. It was concluded that there were negligible internal and external mass transfer resistances, which agreed with the experimental results. In addition, the study of pore diffusion limitations suggested a good agreement between the pseudohomogeneous and detailed heterogeneous models. It can be observed that both models performed similarly when being compared to the experimental data. This indicates the validity and robustness between the models; hence, either model has the ability to predict the catalytic HDO of bio-oil in microreactors. The heterogeneous model has allowed the investigation of pore diffusion limitations by predicting a range of values of the Thiele Modulus at which this occurs. It has shown how this affects the reaction; and gives rise to the study of particle fluid transport phenomena, which aids the understanding of internal and external mass transfer resistances. Performing numerical simulation studies is valuable as it provides an understanding of parameter optimisation and predicting the HDO of other various compounds present in biooil, which would be time consuming and costly to do on an experimental basis.

As the HDO reaction of lignin-derived compounds has not been studied extensively, this model provides a basis to predict and enhance the comprehensive understanding of the HDO reaction in various other lignin-derived compounds in bio-oil. Furthermore, microreactors have demonstrated various benefits ${ }^{11}, 27,28$ compared to conventional reactors, and so future research should be directed towards investigating the scalability of microreactors to be used on an industrial scale.

\section{Conflicts of interest}

There are no conflicts to declare.

\section{Acknowledgements}

The authors would like to thank London South Bank University, School of Engineering, for the PhD funding support.

\section{References}

1. G. Özsin and A. E. Pütün, Energy Conversion and Management, 2017, 149, 675-685.

B. Biswas, N. Pandey, Y. Bisht, R. Singh, J. Kumar and T. Bhaskar, Bioresource technology, 2017, 237, 57-63.

3. N. Joshi and A. Lawal, Industrial \& Engineering Chemistry Research, 2013, 52, 4049-4058. 
737 4. S. Hawash, J. Y. Farah and G. El-Diwani, Journal of Analytical and Applied Pyrolysis, 2017, 124, 369-372.

6. H. Lee, Y.-M. Kim, I.-G. Lee, J.-K. Jeon, S.-C. Jung, J. Do Chung, W. G. Choi and Y.-K. Park, Korean Journal of Chemical Engineering, 2016, 33, 3299-3315.

7. M. Saidi, F. Samimi, D. Karimipourfard, T. Nimmanwudipong, B. C. Gates and M. R. Rahimpour, Energy \& Environmental Science, 2014, 7, 103-129.

8. H. Lee, H. Kim, M. J. Yu, C. H. Ko, J.-K. Jeon, J. Jae, S. H. Park, S.-C. Jung and Y.-K. Park, Scientific reports, 2016, 6, 1-8.

M. L. Patil, A. M. Lali and A. K. Dalai, Asia-Pacific Journal of Chemical Engineering, 2019, 14, e2317.

10. H. Taghvaei and M. R. Rahimpour, Process Safety and Environmental Protection, 2019, 121, 221-228.

11. S. Hafeez, G. Manos, S. Al-Salem, E. Aristodemou and A. Constantinou, Reaction Chemistry \& Engineering, 2018, 3, 414-432.

12. S. G. Newman and K. F. Jensen, Green Chemistry, 2013, 15, 1456-1472.

13. M. D. Subramanyam, A. R. Gollakota and N. Kishore, RSC Advances, 2015, 5, 90354-90366.

14. A. R. Gollakota, M. D. Subramanyam, N. Kishore and S. Gu, RSC Advances, 2015, 5, 41855-41866.

15. Q. Xiong, Y. Yang, F. Xu, Y. Pan, J. Zhang, K. Hong, G. Lorenzini and S. Wang, ACS Sustainable Chemistry \& Engineering, 2017, 5, 2783-2798.

16. Q. Xiong, F. Xu, E. Ramirez, S. Pannala and C. S. Daw, Fuel, 2016, 164, 11-17.

17. G. W. Huber, S. Iborra and A. Corma, Chemical reviews, 2006, 106, 4044-4098.

8. K. Reddy and L. Doraiswamy, Industrial \& Engineering Chemistry Fundamentals, 1967, 6, 77-79.

C. L. Yaws, Journal, 2010.

C. Wilke and P. Chang, AIChE Journal, 1955, 1, 264-270.

C. L. Yaws, Yaws' critical property data for chemical engineers and chemists, Knovel, 2012.

22. J. Richardson, J. Harker and J. Backhurst, Chemical engineering, 2002, 2, 191-236.

S. Fogler, Inc., NJ, 1999.

N. Frössling, Gerlands Beiträge zur Geophysik, 1938, 52, 170-216.

H. Fogler, Journal, 2006.

M. Nowakowska, O. Herbinet, A. Dufour and P.-A. Glaude, The Journal of Physical Chemistry A, 2018, 122, 7894-7909.

27. A. Constantinou, F. Ghiotto, K. F. Lam and A. Gavriilidis, Analyst, 2014, 139, 266-272.

28. X. Sun, A. Constantinou and A. Gavriilidis, Chemical Engineering and Processing: Process Intensification, 2011, 50, 991-997. 